\title{
HMM Parameter Estimation with Genetic Algorithm for Handwritten Word Recognition
}

\author{
Tapan K. Bhowmik ${ }^{1}$, Swapan K. Parui ${ }^{2}$, Manika Kar ${ }^{3}$, and Utpal Roy ${ }^{4}$ \\ ${ }^{1}$ IBM India Pvt Ltd, BCS Building, Salt Lake, Kolkata - 700091, India \\ tkbhowmik@gmail.com \\ ${ }^{2}$ Computer Vision and Pattern Recognition Unit, Indian Statistical Institute, \\ Kolkata-700108, India \\ swapan@isical.ac.in \\ ${ }^{3}$ Cognizant Technology Solutions, Salt Lake, Kolkata - 700091, India \\ manika.kar@cognizant.com \\ ${ }^{4}$ Dept. of Computer and System Sciences, Visva-Bharati, Santiniketan, India \\ roy.utpal@gmail.com
}

\begin{abstract}
This paper presents a recognition system for isolated handwritten Bangla words, with a fixed lexicon, using a Hidden Markov Model (HMM). A stochastic search method, namely, Genetic Algorithm (GA) is used to train the HMM. The HMM is a left-right HMM. For feature extraction, the image boundary is traced both in the anticlockwise and clockwise directions and the significant changes in direction along the boundary are noted. Certain features defined on the basis of these changes are used in the proposed model.
\end{abstract}

\section{Introduction}

Off-line handwritten word recognition is the transcription of handwritten data into a symbolic (ASCII) electronic format. It has several applications such as reading addresses on mail pieces [1, reading amounts on bank checks [2, extracting census data on forms, reading address blocks on tax forms etc. There are two major approaches to recognition of a handwritten word image: analytical approach [3] and holistic approach 44. The idea of analytical approach is to recognize the input word image as a series of segmented sub-images, called primitives. Holistic approach, on the other hand, considers the word image as a single, indivisible entity, and attempts to recognize the word from its over all shape. The present work deals with handwritten word recognition in Bangla with a holistic approach. To the best of our knowledge, no such work is reported on Bangla handwritten word recognition. We consider a set of 117 town names in West Bengal. For feature extraction, the contour of a word image is traced both in clockwise and anticlockwise directions and the points with directional changes are observed. The sequence of such change points represents a basic shape of the word image. Such change points are encoded into certain change codes. These change codes along with their position define the feature vector. A holistic approach based on discrete hidden Markov model (HMM) is used

A. Ghosh, R.K. De, and S.K. Pal (Eds.): PReMI 2007, LNCS 4815, pp. 536-544, 2007.

(C) Springer-Verlag Berlin Heidelberg 2007 
as the recognition engine here. One HMM is constructed for each word class. The Baum-Welch re-estimation method has traditionally been the first choice for training such an HMM. Yet a problem of over fitting on training samples may arise with this method. To resolve this problem to some extent, genetic algorithm (GA) has been used for optimizing the parameters of HMM.

\section{HMM for Word Recognition}

An HMM consists of three sets of parameters $\pi=\left\{\pi_{i}\right\}, A=\left\{a_{i j}\right\}$ and $B=$ $\left\{b_{j k}\right\}, 1 \leq i, j \leq N, 1 \leq k \leq M$, where $\pi$ is the initial state probability distribution, $A$ is the state transition probability distribution matrix and $B$ is the observation symbol probability distribution matrix. Here $N$ is the number of states in the model and $M$ is the total number of distinct observation symbols per state. The elements of the matrices $\left\{a_{i j}\right\}$ and $\left\{b_{j k}\right\}$ always satisfy the following conditions:

$$
\begin{aligned}
& \sum_{j=1}^{N} a_{i j}=1, \text { where } i=1,2, \ldots, N \\
& \sum_{k=1}^{M} b_{j k}=1, \text { where } j=1,2, \ldots, N
\end{aligned}
$$

The complete notation of HMM is $\lambda=(\pi, A, B)$. Each handwritten word is represented by a sequence of observations $O=O_{1}, O_{2}, \ldots, O_{T}$ where $O_{t}$ is the observation symbol observed at time $t$. The isolated word recognition problem can then be regarded as that of computing $\arg \max _{i}\left\{P\left(w_{i} \mid O\right)\right\}$, where $w_{i}$ is the $i^{\text {th }}$ handwritten word. Now using Bayes' rule:

$$
P\left(w_{i} \mid O\right)=\frac{P\left(O \mid w_{i}\right) P\left(w_{i}\right)}{P(O)}=\frac{P\left(O \mid w_{i}\right) P\left(w_{i}\right)}{\sum_{j} P\left(O \mid w_{j}\right) P\left(w_{j}\right)} .
$$

Thus, for a given set of prior probabilities $P\left(w_{i}\right)$, the most probable word depends only on the likelihood $P\left(O \mid w_{i}\right) P\left(w_{i}\right)$. However, for a given $O$ the direct estimation of the joint conditional probability $P\left(O_{1}, O_{2}, \ldots, O_{T} \mid w_{i}\right)$ is not practicable. Instead, for each $w_{i}$, we consider $P\left(O \mid w_{i}\right)=P\left(O \mid \lambda_{i}\right)$, where $\lambda_{i}$ is the HMM corresponding to word $w_{i}$. Thus, in the classification stage, given an unknown input sequence, $O=O_{1}, O_{2}, \ldots, O_{T}$ the probability $P\left(O \mid \lambda_{i}\right)$ is computed for each model $\lambda_{i}$, and $O$ is classified in that class whose model shows the highest likelihood $P\left(O \mid \lambda_{i}\right) P\left(\lambda_{i}\right)$.

\section{Feature Extraction}

Feature extraction is one of the key modules for any recognition system. Several features may be extracted from a word image. But the structural feature is the 
most effective in a problem like the present one. Here the structural feature is extracted as the outer and inner boundaries of a word image traced in both anticlockwise and clockwise directions starting from any boundary point. Suppose such a boundary is represented as $d_{1}, d_{2}, \ldots, d_{n}$, where $d_{i} \in\{1,2, \ldots, 8\}$ is a directional code is shown in Fig. 1. The original image is sufficiently smoothed so that the $e_{i}=d_{i+1}-d_{i}(\bmod 8)$ is +1 or 0 or -1 . Now our goal is to determine pixels where there is a change in direction along the boundary and the type of change. Even in a digital straight line, there may be two different directional codes and hence a change in direction. In order to avoid such spurious changes and find the genuine changes in direction, we do the following. Note that in a digital straight line, $e_{i}$ is always zero except in cases where two consecutive $e_{i}$ values are either $(+1,-1)$ or $(-1,+1)$. Let $E_{j}=\sum_{i=1}^{j} e_{i}$. For any digital straight line, $E_{j}$ is always zero except in cases where it is either -1 or +1 surrounded by zeros. For a genuine change in direction, the value of $E_{j}$ will be non-zero for at least two consecutive pixels. Thus, when for the first time both the values of $E_{j}$ and $E_{j+1}$ are non-zero, we say that there is a change in direction at $(j+1)^{s t}$ pixel. This information of change in direction is encoded as $(D, X, Y)$, where the $D$ represents two consecutive chain codes (Fig. 2) and the coordinates of the $(j+1)^{s t}$ pixel (called a feature point) are given by $(X, Y)$. After a change in direction is encountered, the value of $E_{j}$ is initialized to zero. This process continues until the starting pixel is reached again. This is repeated for both inner and outer boundaries of the image in both clockwise and anticlockwise directions. All types of possible change codes encountered during traversing the image boundary and their corresponding directional codes are shown in Fig. 2,

Note that the change in direction given by the two pairs of directional codes

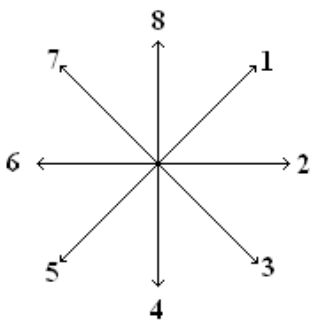

Fig. 1. 8-directional chain codes

$(5,4)$ and $(8,1)$ is the same except that one is obtained from clockwise traversal and the other from the anticlockwise traversal. Thus, these two pairs should result in the same change code. Therefore, all possible changes in direction can be represented by 8 change codes given in Fig. 2. The matrix representation of all possible pairs of directional codes and their corresponding change codes are shown in the Fig. 3. Hence the final feature is $(O, X, Y)$ where $f: D \rightarrow O$, where $O$ is a change code and $f$ is the encoding given by the matrix $\left(c_{i j}\right)_{8 X 8}$ 


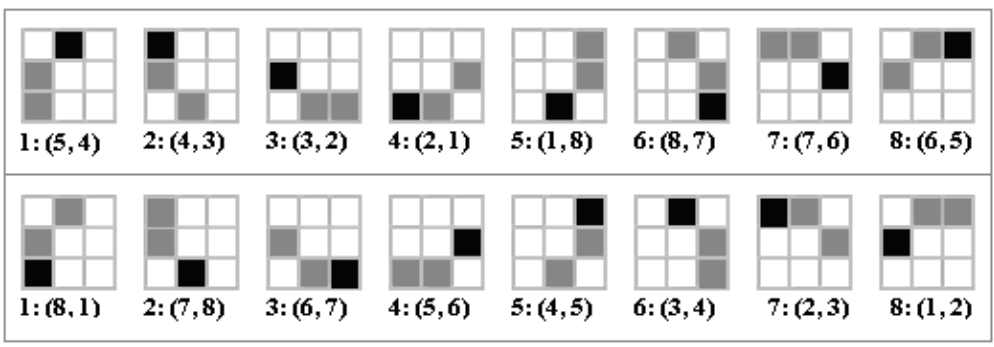

Fig. 2. Change codes corresponding to pairs of directional codes

$$
\left(c_{i j}\right)_{2 \times 8}=\left(\begin{array}{cccccccc}
0 & 8 & 0 & 0 & 0 & 0 & 0 & 5 \\
4 & 0 & 7 & 0 & 0 & 0 & 0 & 0 \\
0 & 3 & 0 & 6 & 0 & 0 & 0 & 0 \\
0 & 0 & 2 & 0 & 5 & 0 & 0 & 0 \\
0 & 0 & 0 & 1 & 0 & 4 & 0 & 0 \\
0 & 0 & 0 & 0 & 8 & 0 & 3 & 0 \\
0 & 0 & 0 & 0 & 0 & 7 & 0 & 2 \\
1 & 0 & 0 & 0 & 0 & 0 & 6 & 0
\end{array}\right)
$$

Fig. 3. Matrix representation of change codes

(Fig. 3) and $(X, Y)$ is the positional information associated with $D$. The contour representations of a word image " $D I G H A$ " as well as the observed feature points when traversed in anticlockwise and clockwise directions are shown in Fig. 4.

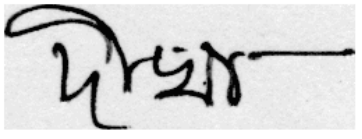

(a)

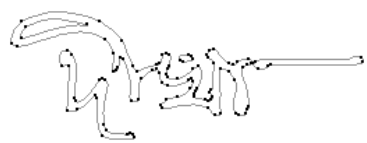

(b)

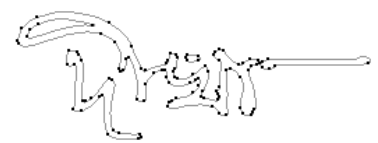

(c)

Fig. 4. (a) Original image (b) Feature points extracted in anticlockwise direction (c) Feature points extracted in clockwise direction

\section{HMM State Definition}

First, we determine the middle zone of the binary word image by analyzing the horizontal histogram. The histogram is drawn based on the following measurement. Let

$$
H_{i}= \begin{cases}\left(\text { row }_{i}-\frac{1}{R} \sum \text { row }_{j}\right), & \text { if }\left(\text { row }_{i}-\frac{1}{R} \sum \text { row }_{j}\right)>0 \\ 0, & \text { otherwise. }\end{cases}
$$


where row $_{i}=$ No of object pixels in the $i^{\text {th }}$ row of the word image, and $R=$ No. of object pixel rows of the word image. The min and max indices of positive $H_{i}$ give the middle zone boundaries of the word image. $H_{i}>0$ indicates that the $i^{\text {th }}$ row is significant. The first significant row from the top and the first significant row from the bottom define the middle zone. To define the states of our proposed HMM, the significant vertical strokes [5] are extracted from the word image. A vertical stroke is a connected component of the set of object pixels whose left neighbors are in the background [5]. The vertical strokes whose number of pixels is greater than (height of the middle zone ${ }^{*} 0.40$ ) are called significant. On the basis of the significant vertical strokes, which are associated with the middle zone, the whole word image is partitioned into several segments as follows. There is a certain segmenting point between each pair of two consecutive vertical strokes. The segmenting point is determined based on the minimum number of object pixels in the vertical histogram of the middle zone between the two vertical strokes. The vertical strokes and their corresponding segmenting points of a few word images from our database are shown in Fig. 5. The vertical strokes are

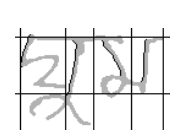

(a)

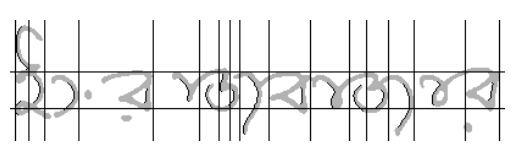

(e)

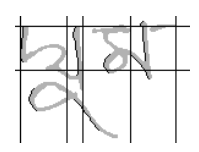

(b)

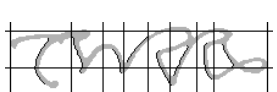

(c)

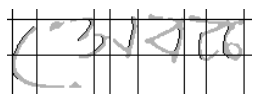

(d)

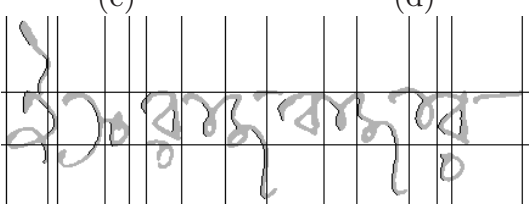

(f)

Fig. 5. Vertical strokes and their corresponding segmenting points for Bangla word images:(a), (b) "GHUM"; (c), (d) "SEBAK" and (e), (f) "ENGRAJBAZAR". The segmenting points are indicated by the vertical straight lines.

arranged from left to right on the basis of $\bar{X}$, where $(\bar{X}, \bar{Y})$ is the centre of gravity of the vertical stroke. Sometimes two consecutive vertical strokes occur very close to each other with one being nearly on the top of the other. In such cases, the larger of the two strokes is retained and the other removed. The strokes are said to be very close if $\left|\bar{X}_{j}-\bar{X}_{j-1}\right| \leq T$, where $T$ is the thickness of the writing pen and $\bar{X}_{j}$ is the $\mathrm{X}$-coordinate of the center of gravity of the vertical stroke $j$. In this study, any three consecutive segments are considered as a single state. Starting from left to right the first state is constructed starting from the first segment. For example, the first state is the triplet consisting of segments $1,2,3$; the second state is the triplet consisting of the segments 2, 3, 4 etc. In Fig [5 (a), the number of segments is 4 , so the number of states is $(4-3+1)=2$. On the other hand, the number of states in Fig 5(c) is $(8-3+1)=6$. In case the number of segments is found to be less than 3 , the whole word image is 
considered as a single state. The distribution of the number of segments for one of the most lengthy words "ENGRAJBAZAR" from our database is shown in Fig 6 where it is seen that the maximum number of segments is found to be 16 .

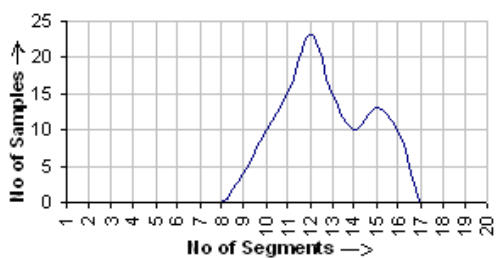

Fig. 6.

So for constructing the HMM for "ENGRAJBAZAR", the number of states is considered $(16-3+1)=14$. In case the number of segments is more than 16 , the last segments are included in the last state $\left(14^{\text {th }}\right.$ state). Similarly, the number of states in the HMM of the word "GHUM" is $(6-3+1)=4$.

\section{Genetic Algorithm}

Genetic algorithms are an optimization technique 6. They have wide use in classification problems for determining the optimal class boundaries, the optimal density parameters etc. The GA is also used in HMM based classification problems [7] for estimating the HMM parameters where a certain fitness function is optimized. The fitness function is always chosen in such a way that the most optimum value of the fitness function gives the best possible HMM in which the input observation sequence most closely fits the HMM model. The basic steps involved in an iteration of GA are described in Table1. Here, $L$ is the population

Table 1. Basic Steps of Genetic Algorithm

\begin{tabular}{|c|c|c|c|c|c|c|}
\hline Gen & $f$ & Prob. & Selection & Pairing & Crossover & Mutation \\
\hline$s_{1}=100 \ldots$ & $f_{1}=f\left(s_{1}\right)$ & $p_{1}=\frac{f_{1}}{F}$ & $s_{1}^{*}$ & $\left(t_{1}, t_{2}\right)$ & $t_{1}^{*}$ & $s_{1}^{* *}$ \\
$s_{2}=001 \ldots$ & $f_{2}=f\left(s_{2}\right)$ & $p_{2}=\frac{f_{2}}{F}$ & $s_{2}^{*}$ & $\left(t_{3}, t_{4}\right)$ & $t_{2}^{*}$ & $s_{2}^{* *}$ \\
$\vdots$ & $\vdots$ & $\vdots$ & $\vdots$ & $\vdots$ & $\vdots$ & $\vdots$ \\
$s_{L}=101 \ldots$ & $f_{L}=f\left(s_{L}\right)$ & $p_{L}=\frac{f_{L}}{F}$ & $s_{L}^{*}$ & $\left(t_{L-1}, t_{L}\right)$ & $t_{L}^{*}$ & $s_{L}^{* *}$ \\
\hline & $F=\sum_{i=1}^{L} f_{i}$ & $\sum_{i=1}^{L} p_{i}=1$ & & & & \\
& & & & & \\
\hline
\end{tabular}

size, $s_{i}, s_{i}^{*}, t_{i}, t_{i}^{*}, s_{i}^{* *}$ are the chromosomes at different stages and $f$ is the fitness function. There are two aspects in any optimization algorithm, namely, exploration and exploitation. The crossover operation is responsible for exploitation while the mutation operation is responsible for exploration in the search space. 


\section{$6 \quad$ HMM Training with GA}

Apart from crossover and mutation operations there are two important components in GA : encoding the chromosome and a fitness function.

\subsection{Encoding Mechanism}

To construct a chromosome, all the parameters of an HMM are arranged in a sequence. In this study, we have used a left-right HMM model with one order jump. A 4-state left-right HMM model with one order jump is shown in Fig 7(a). Note that the initial state distribution here is fixed as $\pi=\{1,0,0, \ldots\}$. Thus $\pi$ is not included in the search space of GA. The observation symbol set here is $\{1,2, \ldots 8\}$ which is the set of change codes shown in Fig 2 . Hence, the number of distinct observation symbols is 8 , but the number of states varies from one HMM to another. For example, the number of states of the HMM for "GHUM" word image is 4 . So the state transition probability distribution is given by $A=\left\{a_{i j}\right\}$ in which there are only $9 a_{i j}>0$, and the other $a_{i j}$ parameters are always 0 $($ Fig $7(b))$.

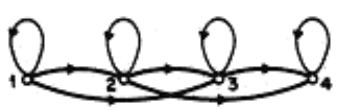

(a)

$$
A=\left[\begin{array}{llll}
a_{11} & a_{12} & a_{13} & 0 \\
0 & a_{22} & a_{23} & a_{24} \\
0 & 0 & a_{33} & a_{34} \\
0 & 0 & 0 & a_{44}
\end{array}\right]
$$

(b)

Fig. 7. (a) LR-HMM model, (b) State transition probability distribution matrix

The observation symbol distribution probability matrix $B=\left\{b_{j k}\right\}$ includes $4 \times 8=32$ parameters where $b_{j k}$ is the probability that the $k^{t h}$ symbol occurs in the $j^{t h}$ state. The total number of parameters of the $\operatorname{HMM} \lambda=(\pi, A, B)$ for "GHUM" word image is $9+32=41$. The training of an HMM involves searching for the best values of these 41 parameters each of which ranges from 0 to 1 . So, in the GA-HMM training, the chromosome consists of two parts, $\mathbf{A}$ and $\mathbf{B}$, one for matrix $A=\left\{a_{i j}\right\}$ and the other for matrix $B=\left\{b_{j k}\right\}$. We encode one chromosome ("GHUM" word image HMM) as 41 real numbers. The genetic representation of the HMM model for the "GHUM" word image is shown in Fig 8 .

\begin{tabular}{|l|l|l|l|l|l|l|l|l|l|l|l|l|}
\hline $\mathrm{A}$ & \multicolumn{1}{|l|}{ B } \\
\hline$a_{11}$ & $a_{12}$ & $a_{13}$ & $a_{22}$ & $\cdots$ & $a_{44}$ & $b_{11}$ & $b_{12}$ & $\cdots$ & $b_{18}$ & $b_{21}$ & $\cdots$ & $b_{48}$ \\
\hline
\end{tabular}

Fig. 8. Genetic representation of HMM for word "GHUM" 


\subsection{Fitness Function}

The fitness function is one of the most crucial components in GA. The objective of the re-estimation process is to find the best possible HMM, which the input observation sequence fits closely. For a given model $\lambda, P(O \mid \lambda)$ measures how far the input observation sequence $O=O_{1}, O_{2}, \ldots$ fits the model. A greater value of $P(O \mid \lambda)$ gives a closer fit. From this point of view, $P(O \mid \lambda)$ has been used in the fitness function. Note that $L$ is the number of solutions in the population and $\lambda^{(i)}$ is the $i^{t h}$ solution of $\lambda$. Then the fitness function is defined as $f_{i}=\frac{q_{i}}{\sum_{l=1}^{L} q_{l}}$, where $q_{i}=\sum P\left(O / \lambda^{(i)}\right)$ is the sum of probabilities of all the training observations in a word class for a given $\lambda^{(i)} . P\left(O \mid \lambda^{(i)}\right)$ has been calculated by the well-known forward algorithm 8 .

\subsection{Initial Gene Production}

For every sample image in a particular word class, a set of feature vectors of the form $(O, X, Y)$ is extracted, where $O$ is a change code and $(X, Y)$ is the position of the corresponding feature point. Let this set be $\left(O_{i}, X_{i}, Y_{i}\right), i=1,2, \ldots T$. Suppose the number of states is $N$. Each $O_{i}$ will belong to one state or two states or three states depending on $\left(X_{i}, Y_{i}\right)$. For example, if $\left(X_{i}, Y_{i}\right)$ falls in segment 2, then $O_{i}$ will belong to both the states 1 and 2. This is repeated for all the training images of a particular word class. For each state, $b_{j k}$ is computed as the relative frequency of the $k^{t h}$ change code in the $j^{t h}$ state. $B=\left\{b_{j k}\right\}$ is the initial estimate of the observation symbol probability distribution, which will be incorporated in each of $L$ chromosomes. Now, no initial estimates are made for $A=\left\{a_{i j}\right\}$. Random numbers are generated to compute the initial values of $a_{i j}$ 's such that $\sum_{j} a_{i j}=1$, for all $i$. In this experiment, population size $L$ has been taken to be 20 .

\subsection{Crossover and Mutation}

For GA-HMM training, roulette wheel selection scheme has been used to select the chromosome. After selection is over, pairs of chromosomes are picked up randomly from the population to be subjected to crossover. In this study double point crossover mechanism is used. Two points are chosen randomly, one is from part $\mathbf{A}$ and the other is from part $\mathbf{B}$. The portions of two chromosomes between two selected crossover points are exchanged to reproduce two new chromosomes. For mutation operation one chromosome is chosen randomly from the population. From it, two bits, one from part $\mathbf{A}$ and the other from part $\mathbf{B}$, are selected randomly to be flipped. Both the crossover and mutation operations are controlled by two probabilities: crossover probability $\rho_{c}$ and mutation probability $\rho_{m}$. For training an HMM we have used $\rho_{c}=0.7$ and $\rho_{m}=0.01$. It should be noted that after crossover or mutation, all the HMM parameters of the population are normalized to satisfy the equations 1 and 2 . 


\section{$7 \quad$ Experimental Results}

The experiment has been carried out on a recently developed database of handwritten Bangla words. We have considered 117 town names in West Bengal. Our database contains 14,625 handwritten word samples from 125 writers. Each writer has written only one sample word for each town name. Then it was randomly divided into training and test sets. The training set consists of 11700 word image samples while the test set consists of 2925 word image samples. The recognition accuracy found on test set varies between $71 \%$ to $86 \%$.

\section{Conclusion}

The proposed method is quite general in the sense that it can be applied to other scripts also. Only the state definition is to be changed. Another aspect is that no size normalization is necessary in the proposed approach. Also, it is robust with respect to minor rotations of the input image.

\section{References}

1. Dehghan, M., Faez, K., Ahmadi, M., Shridhar, M.: Unconstrained farsi (arabic) handwritten word recognition using fuzzy vector quantization and hidden markov models. Pattern Recognition Letters 22, 209-214 (2001)

2. Guillevic, D., Suen, C.: Hmm word recognition engine. In: Proceedings of the Int. Conf. on Document Analysis and Recognition, pp. 544-547 (1997)

3. Govindaraju, V., Kim, G.: A lexicon driven approach to handwritten word recognition for real-time applications. IEEE Trans. Pattern Analysis Machine Intelligence 19(4), 366-379 (1997)

4. Madhvanath, S., Govindaraju, V.: The role of holistic paradigms in handwritten word recognition. IEEE Trans. Pattern Analysis Machine Intelligence 23(2), 149$164(2001)$

5. Bhowmik, T., Bhattacharya, U., Parui, S.: Recognition of bangla handwritten characters using an mlp classifier based on stroke features. In: Pal, N.R., Kasabov, N., Mudi, R.K., Pal, S., Parui, S.K. (eds.) ICONIP 2004. LNCS, vol. 3316, pp. 814-819. Springer, Heidelberg (2004)

6. Goldberg, D.: Genetic algorithms in search optimization and machine learning. Addison-Wesley, Reading (1989)

7. Won, K., Prugel-Bennett, A., Krogh, A.: Training hmm structure with genetic algorithm for biological sequence analysis. Bioinformatics 20(18), 3613-3619 (2004)

8. Rabiner, L.: A tutorial on hidden markov models and selected applications in speech recognition. Trans. on. IEEE 77(2), 257-286 (1989) 